\title{
Vom Sinn und Unsinn ophthalmologischer Eponyme
}

\section{Sense and Nonsense of Ophthalmological Eponyms}

„Eponyme bringen Farbe in die Medizin, und sie betten medizinische Traditionen und Kultur in unsere Geschichte ein“. (Judith Whitworth, australische NationalUniversität Canberra) [1].

Unter einem Eponym versteht man die wissenschaftliche Bezeichnung mittels eines Namensgebers, der zumeist dem Erstbeschreiber oder Erfinder entspricht, also z.B. „Axenfeld-Anomalie“ (nach Theodor Axenfeld, 1867-1930) [2], „Kayser-Fleischer-Ring“ (nach Bernhard Kayser, 1869-1954, und Bruno Fleischer, 1874-1965) [3], „Elschnig-Perlen“ (nach Anton Elschnig, 1863-1939) [4] ( $\triangle$ Abb. 1, 2 und 3), „Graefe-Zeichen“ (nach Albrecht von Graefe, 1828-1870) oder "Sturge-Weber-Syndrom" (nach William Allen Sturge, 1850-1919, und Frederick Parkes Weber, 1863-1962, beide keine Ophthalmologen) $[5,6]$. Rechnet man die zahlreichen „Geräte-Eponyme“ wie z.B. „Jäger-Tafeln“ (nach Eduard von Jäger, 1818-1884), „Hertel-Exophthalmometer“ (nach Ernst Hertel, 1870-1943), „Amsler-Netz“ (nach Marc Amsler, 18911968) oder „Sautter-Kanüle“ (nach Hans Sautter, 1912-1984) hinzu, so dürfte es mehrere hundert Eponyme geben, die das augenärztliche Fachgebiet unmittelbar oder zumindest am Rande betreffen. Eine zusammenfassende Darstellung aller derzeit geläufigen ophthalmologischen Eponyme gibt es nicht. Vor bald 50 Jahren hat Stephan von Grósz aus Budapest die

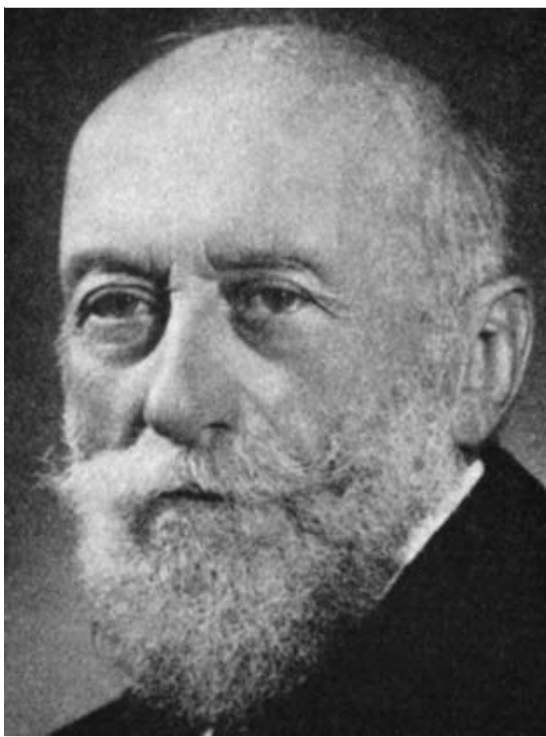

Abb. 1 Anton Elschnig, Namensgeber der „Elschnig-Perlen“. Elschnig wurde vor 75 Jahren (1939) als Fußgänger auf dem Wiener Ring von einem $\mathrm{Kfz}$ erfasst. Er starb noch am Unfallort. Bild aus dem Nachruf (Klin Monatsbl Augenheilkd 1940; 104: 101-104).

seinerzeit bekannten syndromalen Eponyme zusammengestellt [7]. Im Internet stößt man auf „Doyne's Hall of Fame -

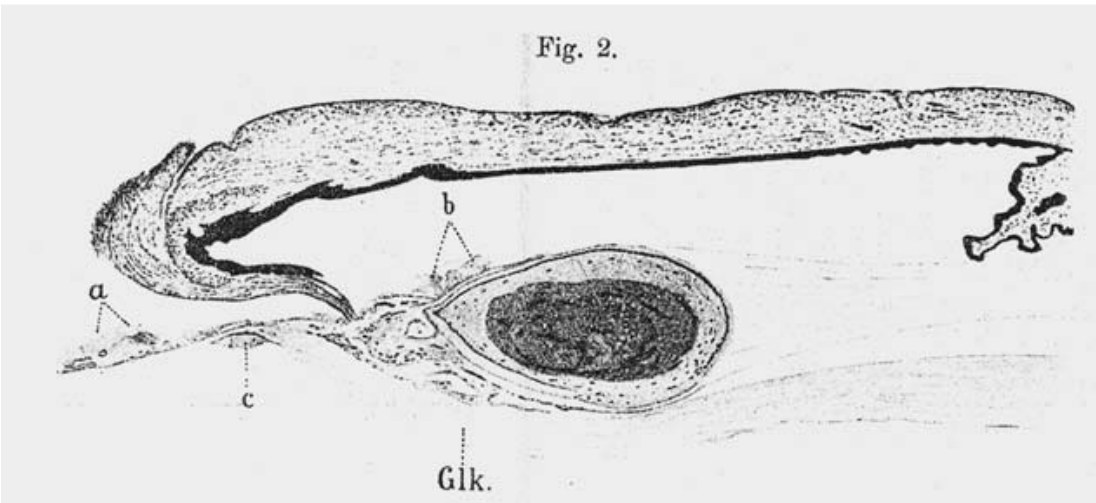

Abb. 2 Pathohistologische Darstellung des regeneratorischen Nachstars aus Elschnigs Originalpublikation von 1911 [4].

hinteren Kapsel auf (s. Fig. 2 a, Fig. 3a), teils sind sie anscheinend zu kompakteren, dann immer gleichmässig bläulich gefärbten Gebilden zusammengeflossen; an einzelnen Stellen liegen sie fast kugelig und deutlich abgegrenzt, dann gewöhnlich etwas dunkler blau gefärbt, den mehr kompakten Grundschichten auf und prominieren stark gegen die Pupille zu. Nur an wenigen Stellen ist diese Auflagerung von der Kapsel durch

Fig. 3.

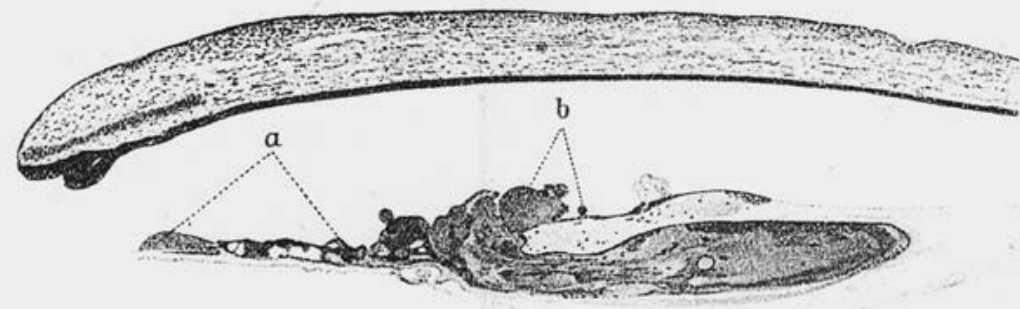




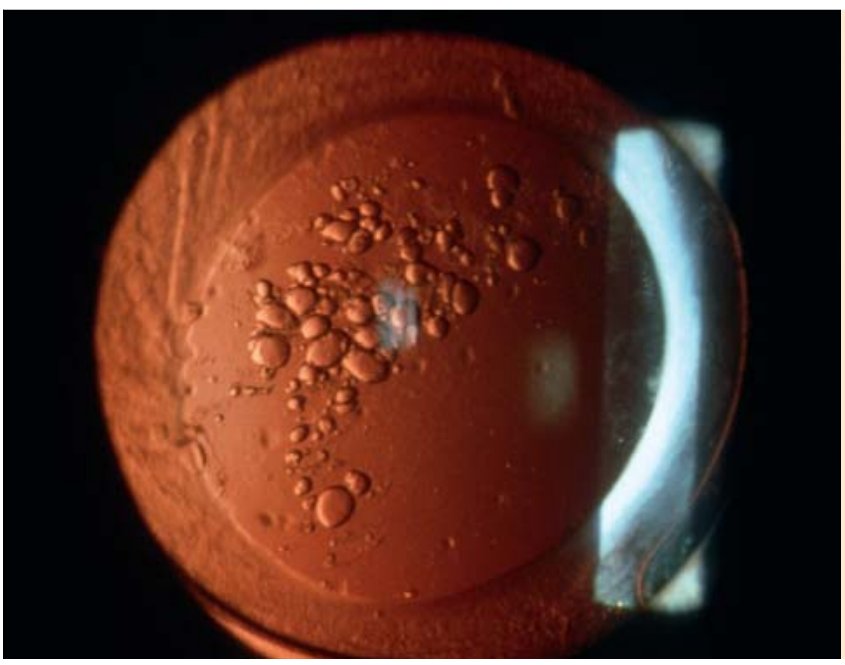

Abb. 3 Regeneratorischer Nachstar („Elschnig-Perlen“) bei Pseudophakie im regredienten Licht (UniversitätsAugenklinik Tübingen).

Faces behind ophthalmological eponyms“ mit 85 Namen (Stand November 2014). Eponyme haben in allen medizinischen Fachgebieten - so auch der Ophthalmologie - eine lange Tradition. Nichtsdestotrotz ist ihre Sinnhaftigkeit seit der Jahrtausendwende, insbesondere in den USA und auf dem Gebiet der inneren Medizin, zunehmend infrage gestellt worden.

Argumente „pro Eponym“ waren und bleiben [1]:

- Der Erstbeschreiber wird geehrt.

- Durch die Assoziation mit einem Leben ist eine zeitliche Einordnung des Eponyms bzw. der Erstbeschreibung möglich. So kann z.B., wenn man die Lebensdaten Theodor Axenfelds ungefähr kennt, die Beschreibung der AxenfeldAnomalie auf „grob Anfang des 20. Jahrhunderts“ datiert werden (Die Erstbeschreibung erfolgte 1920 im Rahmen eines Vortrags bei der 42. Tagung der DOG in Heidelberg [2]).

- Das Eponym ist in aller Regel kürzer und griffiger als die pathologische, pathophysiologische oder pathogenetische Bezeichnung. So wäre es höchst umständlich, wenn z.B. statt „RiegerAnomalie“ (nach Herwigh Rieger, 1898-1986) [8] stets „Dysgenesis mesodermalis iridis et cornea“ gesagt oder geschrieben werden müsste.

Auf der anderen Seite gibt es gewichtige Gründe „contra Eponym“ [9].

- Das Eponym ist „unspezifisch“, da es nicht das pathologische, pathophysiologische oder pathogenetische Charakteristikum der Erkrankung wiedergibt.

- Das Eponym ist nicht selten „ungerecht“, weil die Priorität der Erstbeschreibung oft nicht klar ist und „substanzielle Mitbeschreiber“ vergessen werden. So heißt, um ein Beispiel zu nennen, die (klinisch völlig unbedeutende) Pigmentlinie auf dem Hornhauttransplantat üblicherweise „Mannis-Linie“, weil Mark Mannis diese Eisenlinie 1983 bei einer etwas größeren Serie von 8 Patienten beschrieb (und dabei feststellte, dass die Linie frühestens 8 Wochen nach perforierender Keratoplastik zu sehen ist) [10]. Beschrieben wurde die Linie aber schon 2 Jahre zuvor von Forstot und Abel wenngleich nur in einem kurzen Abstract [11]. „Gerechter“ wäre also das Eponym „Forstot-Abel-Mannis-Linie“. Die nach Louis Braille (1809-1852) benannte Blindenschrift wurde eigentlich von dem französischen Artilleriehauptmann Charles Barbier (1767-1841) entwickelt, damit seine Befehle von seinen Soldaten auch bei Nacht („Nachtschrift“) aber nicht vom Feind („Geheimschrift“) gelesen werden konnten [12]. Die Punktschrift, die Braille vereinfachte und praktikabel machte, müsste daher eigentlich Barbier-Braille-Schrift heißen. Und erst jüngst wies Hans-Reinhard Koch nach intensivem Studium alter Literatur in seinem Vortrag „Giovanni Baptista Morgagni und sein Humor" anlässlich des 112. Kongresses der DOG in Leipzig darauf hin, dass Morgagni (1682-1771) sehr wahrscheinlich nicht die eigentliche „Cataracta hypermatura Morgagni“ mit dem im Kapselsack abgesunkenen Linsenkern sondern nur ,weiche Rinde" beschrieben hat.

- Für die gleiche Erkrankung werden, je nach Land, unterschiedliche Eponyme gebraucht, was die „internationale Verständigung“ erschwert. Bestes Beispiel ist wohl die endokrine Orbitopathie, die bei uns zum „Morbus Basedow“ (nach Carl Adolph von Basedow, 1799-
1854), im angloamerikanischen Raum aber zur „Graves disease“ (nach dem irischen Kliniker Robert James Graves, 1797-1853) gehört. In Frankreich wird die endokrine Orbitopathie gar mitunter als „Maladie de Demours“ (nach Antoine Pierre Demours, 1762-1836) bezeichnet [13].

- Einige Eponymgeber sind „politisch belastet“. Dieses gilt praktisch nur für deutsche Erstbeschreiber, die während der Zeit des Nationalsozialismus Mitglied der NSDAP oder einer ihrer Gliederungen waren. Beispielhaft kann die Debatte um die „Wegener'sche Granulomatose" (nach dem Pathologen Friedrich Wegener, 1907-1990) angeführt werden, die nach einem Lancet-Beitrag von 2006, in dem auf Wegeners Parteiund SA-Mitgliedschaft hingewiesen wurde [14], vor allem in den USA sehr heftig geführt wurde. Dabei blieben Friedrich Wegener entlastende Momente großenteils unberücksichtigt [15]. Als Konsequenz der Debatte wurde der „Morbus Wegener“ nach einer Konsensus-Konferenz namhafter Rheumatologen 2010 in „Granulomatose mit Polyangiitis/GPA (Wegener)“ umbenannt, wobei die Klammer mit „Wegener“ nach einer Übergangszeit von 5 Jahren entfallen soll [16]. Damit wird das Eponym „Wegener“ 2015/2016 „formal sterben“. Ob sich die Umbenennung tatsächlich durchsetzen wird, bleibt abzuwarten. In der Augenheilkunde haben wir derzeit noch 5 Entitäten bzw. Prozeduren, die nach einem (nach bisherigem Forschungsstand nicht in NS-Verbrechen verwickelten) „Parteigenossen“ benannt sind.

Die Diskussion um die medizinischen Eponyme hält an, verläuft in der deutschen Augenheilkunde bisher aber noch „subkutan“. Die neuesten „Kreationen“ auf ophthalmologischem Fachgebiet dürften das „Flammer-Syndrom“ (nach Josef Flammer aus Basel) und die prädescemetäre, morphologisch nicht wirklich fassbare und daher fragwürdige „DuaSchicht" (nach Harminder Dua aus Nottingham) sein. Spannend wird bleiben, ob und inwieweit sich die eher Eponymfreundlichen Europäer gegen die eher Eponym-reservierten Amerikaner z.B. bei der neuen Klassifikation der noch oft mit einem Eponym belegten Hornhautdystrophien (HD) - man denke nur an die „Meesmann-HD“ (nach Alois Meesmann, 1888-1969) [17] oder die „ThielBehnke-HD“ (nach Hans-Jürgen Thiel und Horst Behnke) [18] - durchsetzen 
werden. Der Kompromiss könnte darin bestehen, dass man es bei den in der Literatur eingeführten Eponymen belässt, bei neuen Entitäten aber auf ein Eponym verzichtet und eine pathologische, pathophysiologische, pathogenetische oder deskriptive Bezeichnung wählt.

\section{Interessenkonflikt: Nein.}

\section{J. M. Rohrbach}

Department für Augenheilkunde,

Forschungsbereich Geschichte der Augenheilkunde/Ophthalmopathologisches Labor, Eberhard-Karls-Universität Tübingen

\section{Literatur}

1 Whitworth JA. Should eponyms be abandoned? No. Br Med J 2007; 335: 425

2 Axenfeld T. Embryotoxon corneae posterius. Klin Monatsbl Augenheilkd 1920; 65: 381382

3 Lang GK. 1902/1903, Bernhard Kayser und Bruno Fleischer. Klin Monatsbl Augenheilkd 2013; 230: 310-312

4 Elschnig A. Klinisch-anatomischer Beitrag zur Kenntnis des Nachstares. Klin Monatsbl Augenheilkd 1911; 49: 444-451

5 Sturge WA. A case of partial epilepsy, apparently due to a lesion of one of the vaso-motor centres of the brain. Clin Soc Transact 1879; $12: 162$

6 Weber FP. Right-sided hemi-hypotrophy resulting from right-sided congenital spastic hemiplegia, with a morbid condition of the left side of the brain, revealed by radiograms. J Neurol Psychopathol 1922; 37: 184-189

7 von Grósz S. Eponyme - Syndrome. An Autorennamen gebundene augenärztliche Symptomenkomplexe und Krankheiten. Klin Monatsbl Augenheilkd 1966; 148: 1-45

8 Rieger $H$. Beiträge zur Kenntnis seltener Mißbildungen der Iris. II. Über Hypoplasie des Irisvorderblattes mit Verlagerung und Entrundung der Pupille. Graefes Arch Clin Exp Ophthalmol 1935; 133: 602-635

9 Woywodt A, Matteson E. Should eponyms be abandoned? Yes. Br Med J 2007; 335: 424

10 Mannis MJ. Iron deposition in the corneal graft. Arch Ophthalmol 1983; 101: 18581861

11 Forstot SL, Abel R. Epithelial iron line developing in the donor cornea postkeratoplasty. Ophthalmology (Suppl) 1981; 88: 88

12 Rohrbach JM, Dzhelebov DN, Neuhann I. Emile Javal (1839-1907) und seine GlaukomErblindung - ein Leben für Politik, Publizistik, sinnesphysiologische Forschung und Erblindete. Klin Monatsbl Augenheilkd 2011; 228: 166-169

13 Göring H-D. Carl Adolph von Basedow (1799-1854). Eine Krankheit trägt seinen Namen. Dtsch Ärztebl 2014; 111: B-548549

14 Woywodt A, Haubitz M, Haller H et al. Wegener's granulomatosis. Lancet 2006; 367 : 1362-1366

15 Grzybowski A, Rohrbach JM. Sollten wir auf das Eponym „Wegener'sche Granulomatose" verzichten? Ein historischer Exkurs. Klin Monatsbl Augenheilkd 2011; 228: 641-643
16 Falk RJ, Gross WL, Guillevin L et al. Granulomatosis with polyangiitis (Wegener's): An alternative name for Wegener's granulomatosis. Arthritis \& Rheumatism 2011; 63: 863-864

17 Meesmann A. Über eine bisher nicht beschriebene, dominant vererbte Dystrophia epithelialis corneae. Bericht über die zweiundfünfzigste Zusammenkunft der DOG. München: Bergmann 1938: 154-158

18 Thiel H-J, Behnke $\mathrm{H}$. Eine bisher unbekannte subepitheliale hereditäre Hornhautdystrophie. Klin Monatsbl Augenheilkd 1967; 150: 862-874

\section{Bibliografie}

Dol http://dx.doi.org/10.1055/s-0034-1383385 Online-publiziert: 15.12.2014

Klin Monatsbl Augenheilkd 2015; 232: 193-195

(c) Georg Thieme Verlag KG Stuttgart · New York . ISSN 0023-2165

\section{Korrespondenzadresse}

\section{Prof. Dr. Jens Martin Rohrbach}

Department für Augenheilkunde, Forschungs-

bereich Geschichte der Augenheilkunde/

Ophthalmopathologisches Labor

Eberhard-Karls-Universität Tübingen

Schleichstr. 6-12

72076 Tübingen

Tel.: + 49/(0)7071/2984761

Fax: + 49(0)7071/294762

Martin.Rohrbach@med.uni-tuebingen.de

\section{License terms}

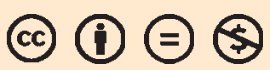

